\title{
A NOTE ON QUASI AND BI-IDEALS IN TERNARY SEMIGROUPS
}

\author{
V. N. DIXIT and SARITA DEWAN \\ Department of Mathematics \\ Unlvorglty of Dolhi \\ Delhi \\ India
}

(Received May 11, 1993 and in revised form November 3, 1993)

ABSTRACT. In this paper we have studied the properties of Quasi-ideals and $\mathrm{Bi}-$ ideals in ternary semi groups. We prove that every quasi-ideal is a bi-ideal in $T$ but the converse is not true in general by giving several example in different context.

KEY WORDS AND PHRASES. Quasi-ideal, Bi-ideal, Ternary Semi group. 1992 AMS SUBJECT CLASSIFICATION CODE 20N99

\section{INTRODUCTION.}

D.H. Lehmer [4] gave the definition of a ternary semi group as follows:

DEEINITION 1.1. A non-empty set $T$ is called a ternary semigroup if a ternary operation [ ] on $\mathrm{T}$ is defined and satisfies the associative law

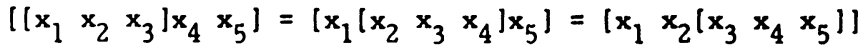

for all $x_{i} \in T, 1 \leq 1 \leq 5$.

Banach showed by an example that a ternary semi group does not necessarily reduce to an ordinary semi group. This has been shown by the following example.

EXAMPLE 1.2. Let $T=\{-i, 0, i\}$ be a ternary semi group under the multiplication over complex number while $\mathrm{T}$ is not a binary seml group under the multiplication over complex number.

Los [5] showed that any ternary semi group however may be embedded in an ordinary semi group in such a way that the operation in the ternary semi group is an (ternary) extension of the (binary) operation of the containing semi broup.

Dudek [1], Feizullaer [2], Kim and Roush [3], Lyapin [6] and Sioson [7] has also studied the properties of the ternary semi groups.

We give the following definitions of ideals [7] as follows:

DEFINITION 1.3. A left (right, lateral) ideal of a ternary semi group $T$ is a non-empty subset $L(R, M)$ of $T$ such that 


$$
[T T L] \subseteq L([R T T] \subseteq R,[T M T] \subseteq M)
$$

DEFINITION 1.4. If a non-empty subset of $\mathrm{T}$ is a left, right and lateral ideal of $T$, then it is called an ideal of $T$.

DEFINITION 1.5. For each element $t$ in $T$, the left, right and lateral ideal generated by ' $t$ ' are respectively given by:

$$
\begin{aligned}
& (t)_{L}=\{t\} \cup[\text { TTt }] \\
& (t)_{R}=\{t\} \cup[t T T] \\
& (t)_{M}=\{t\} \cup[\text { TtT] }][\text { TTtTT] }
\end{aligned}
$$

Due to associative law in $T$, one may write Sioson [7]

$$
\begin{aligned}
{\left[\begin{array}{ll}
x_{1} & x_{2} \cdots x_{2 n+1}
\end{array}\right] } & =\left[x_{1} \cdots x_{m m+1} x_{m+4} \cdots x_{2 n+1}\right], m \leq n \\
& =\left[x_{1} \ldots\left[\left[x_{m} x_{m+1} x_{m+2}\right] x_{m+3} x_{m+4}\right] \cdots x_{2 n+1}\right], m \leq n
\end{aligned}
$$

DEFINITION 1.6. Quasi-ideal in a ternary semi group [7] is also a subset $Q$ of $T$ (possibly empty) satisfying following two conditions:

(1) $[$ QTT $] \cap[T Q T] \cap[T T Q] \subseteq Q$

(2) [QTT] $\cap[$ TTQTT $] \cap[T T Q] \subseteq Q$

REMARK 1.7. Every right, left and lateral ideal is a quasi-ideal. But every quasi-ideal is not a right, a left and a lateral ideal of $T$. This follows from the following example

EXAMPLE 1.8. Let $T=\left\{\left(\begin{array}{ll}0 & 0 \\ 0 & 0\end{array}\right),\left(\begin{array}{ll}1 & 0 \\ 0 & 0\end{array}\right),\left(\begin{array}{ll}0 & 1 \\ 0 & 0\end{array}\right),\left(\begin{array}{cc}0 & 0 \\ 1 & 0\end{array}\right),\left(\begin{array}{ll}0 & 0 \\ 0 & 1\end{array}\right)\right\}$ be the ternary semi group under matrix multiplication. Then $Q=\left\{\left(\begin{array}{ll}0 & 0 \\ 0 & 0\end{array}\right),\left(\begin{array}{ll}0 & 1 \\ 0 & 0\end{array}\right)\right\}$ be the quasiideal of $T$, which is neither a left, nor a right nor a lateral ideal of $T$.

DEFINITION 1.9. A ternary sub semi group is a subset $S$ of a ternary semi group T such that

$$
[\mathrm{SSS}] \subseteq \mathrm{S}
$$

DEFINITION 1.10. A ternary semi group $T$ is said to be a ternary group if it satisfies the following property that for all $x, y$ and $z$ in $T$, there exists unique $a, b, c$ in $T$ such that

$$
[x a b]=c,[a y b]=c,[a b z]=c
$$

DEEINITION 1.11. A ternary group $T$ is said to be a ternary group with 0 if for all $a, b, c$ in $T$

$$
[o a b]=0=[a o b]=[a b o]=[a o o]=[o b o]=[o o c] \text {. }
$$

DEFINITION 1.12. A ternary semi group $T$ is with identity if there exists an 
idempotent $\mathrm{e}$ in $\mathrm{T}$ such that

$$
[\mathrm{aae}]=[\text { eaa }]=[\mathrm{aea}]=\mathbf{a}, \forall \mathrm{a} \in \mathrm{T} \text {. }
$$

2. SOME RESULTS ON QUASI-IDEAL IN T WHICH ARE TRIVIALLY TRUE

PROPOSITION 2.1. A ternary group $T$ with 0 and [TTT] $\neq 0$ has no proper quasiideal.

PROPOSITION 2.2. The intersection of a quasi-ideal $Q$ and a ternary sub semigroup $A$ of a ternary semi group $T$ is either empty or a quasi-ideal of $A$.

PROPOSITION 2.3. Let $Q$ be any non-empty subset of aternary semi group $T$, then the following are true:

(1) $Q \cup[T T Q]$ is the smallest left ideal of $T$ containing $Q$.

(2) $Q \cup[Q T T]$ is the smallest right ideal of $T$ containing $Q$.

(3) $Q \cup[T Q T] \cup$ [TTQTT] is the smallest lateral ideal of $T$ containing $Q$.

(4) If $Q$ is a quasi-ideal of $T$. Then

$Q=(Q \cup[T T Q])$ i $(Q \cup[T Q T] \cup[T T Q T T]) \cap(Q \cup[Q T T])$.

PROPOSITION 2.4. The intersection of arbitrary set of quasi-ideals in a ternary semi group is either empty or a quasi-ideal of $T$.

DEFINITION 2.5. Let $X$ be a non-empty subset of a ternary semi group $T$. The quasi-ideal of $T$ generated by $X$ is intersection of all quasi-ideals $(X)_{q}$ of $T$ containing $X$.

If the subset $X$ consists of a single element $X$, then $(X)_{q}$ is the cyclic quasiideal of $\mathrm{T}$.

PROPOSITION 2.6. Let $X$ be a non-empty subset of ternary semi group $T$, then

$$
(X)_{q}=(X \cup[T T X]) \cap(X \cup[T X T] \cup[T T X T T]) \cap(X \cup[X T T])
$$

is the smallest quasi-ideal containing $X$.

PROOF. Sioson [7] shows that the intergection of a right, a left and a lateral ideal of a ternary semi group $T$ is a quasi-ideal. Therefore the proof easily follows by using 2.3 .

From 2.6 it follows that

$$
(X)_{q}=(\{X\} \cup \underline{.}[\operatorname{TTX}]) n(\{X\} \cup[T X[] \cup[\operatorname{TTX}[T]), n(\{X\} \cup[X[T])
$$

is the smallest quasi-ideal of $T$ containing $X$.

3. BI-IDEALS IN TERNARY SEMI GROUP

DEEINITION 3.1. A ternary sub semi group $B$ of a ternary semi group $T$ is a biideal of $\mathrm{T}$ if $[$ (BTBTB] $\subseteq \mathrm{B}$.

PROPOSITION 3.2. Every quasi-ideal of a ternary semi group $T$ is a bi-ideal. 
PROOF. Let $Q$ be a quasi-ideal of $T$. Then $Q$ is a ternary semi group of $T$. Now [RTRTQ] $\leq[Q[T T T] T] \subseteq[Q T T]$.

Similarly [QTOQTQ] $\subseteq[T T Q] \cap$ [TTQTT].

Therefore [QTQTQ] $\subseteq[T T Q] \cap[T T Q T T] \cap[Q T T] \subseteq Q$.

PROPOSITION 3.3. Let $A$ be an ideal and $Q$ be a quasi-ideal of $T$. Then $A \cap Q$ is a bi-ideal and a quasi-ideal of $\mathrm{T}$.

PROOF. [ $A \cap Q \quad A \cap Q \quad A \cap Q] \subseteq[A A A] \cap[Q Q Q] \subseteq A \cap Q$ implies that $A \cap Q$ is a ternary sub semi group of $T$. Also

$$
[A \cap Q T A \cap Q T A \cap Q] \subseteq[Q T Q T Q] \cap[A[T A T \not A \exists \leq Q \cap[A A A]
$$

by (3.2) and the given hypothesis implies that L.H.S. $\subseteq Q \cap A$. Thus An $Q$ is a biideal of $T$. Since $A$ is an ideal of $T$ and it is also a quasi-ideal of $T$. Hence $A \cap Q$ is a quasi-ideal of $\mathrm{T}$.

PROPOSITION 3.4. Let $X, Y$ be non-empty subsets of ternary semi group $T$, then $N=[X T Y]$ is a bi-ideal of $T$.

PROOF. Clearly $\mathrm{N}$ is a ternary sub semi group of $\mathrm{T}$. Also

$$
\begin{aligned}
& {[\text { NTNTN }] \subseteq[X[T T T][T T T][T T T] Y] \subseteq[X[T T T] Y] } \\
& \subseteq[X T Y]=N .
\end{aligned}
$$

Then $\mathrm{N}$ is a bi-ideal of $\mathrm{T}$.

PROPOSITION 3.5. The intersection of arbitrary set of bi-ideals of $T$ is either empty or a bi-ideal of $\mathrm{T}$.

We omit the trivial proof.

PROPOSITION 3.6. Every left, right or lateral ideal of $T$ is a bi-ideal of $T$.

PROOF. Trivial.

PROPOSITION 3.7. Let $Q$ be a subset of a ternary seml group $T$ and $Y$ be a nonempty proper subset of $T$ such that

(1) [TTQ] $\cup[T Q T] \cup[Q T T] \cup[T T Q T T] \subseteq Y$.

(2) $Y \subseteq Q$.

Then $Y$ is an ideal of $T$. Moreover $Y$ is a bi-ideal of $T$.

PROOF. It is obvious that [TTY], [TYT]. [YTT] and [TTYTT] are contained in $Y$ under the condition (2) therefore $Y$ is an ideal of $T$. And hence a quasi-ideal of $T$ which by 3.2 is a bi-ideal of $\mathrm{T}$.

In the following example we show that if both or either of the conditions (I) and (2) of above proposition are not satisfied then $Y$ is neither a left, a right, a lateral, a quasi nor a bi-ideal of $T$.

EXAMPLE 3.8. Let $\mathrm{T}=\left\{\left(\begin{array}{ll}0 & 0 \\ 0 & 0\end{array}\right),\left(\begin{array}{ll}1 & 0 \\ 0 & 1\end{array}\right),\left(\begin{array}{ll}1 & 0 \\ 0 & 0\end{array}\right),\left(\begin{array}{ll}0 & 1 \\ 0 & 0\end{array}\right),\left(\begin{array}{ll}0 & 0 \\ 1 & 0\end{array}\right),\left(\begin{array}{ll}0 & 0 \\ 0 & 1\end{array}\right)\right\}$. Then $T$ is a ternary semi group under matrix multiplication. 
(1) Take $Y=\left\{\left(\begin{array}{ll}0 & 0 \\ 0 & 0\end{array}\right),\left(\begin{array}{ll}1 & 0 \\ 0 & 1\end{array}\right)\right\}$ and the quasi-ideal

$$
Q=\left\{\left(\begin{array}{ll}
0 & 0 \\
0 & 0
\end{array}\right),\left(\begin{array}{ll}
1 & 0 \\
0 & 0
\end{array}\right)\right\} \text { of } \mathrm{T} \text {. }
$$

We see that $Y \nsubseteq Q$, and

$$
\begin{aligned}
& {[T T Q] \cup[T Q T] \cup[\operatorname{TTQTT}] \cup[Q T T]} \\
& =\left\{\left(\begin{array}{ll}
0 & 0 \\
0 & 0
\end{array}\right),\left(\begin{array}{ll}
1 & 0 \\
0 & 0
\end{array}\right),\left(\begin{array}{ll}
0 & 0 \\
1 & 0
\end{array}\right),\left(\begin{array}{ll}
0 & 1 \\
0 & 0
\end{array}\right),\left(\begin{array}{ll}
0 & 0 \\
0 & 1
\end{array}\right)\right\} \\
& \notin Y
\end{aligned}
$$

Also each of [TTY], [TYT], [YTT] and [TTYTT] is not in Y.

Therefore $Y$ is neither a left, nor a right nor a lateral ideal of $T$.

Moreover [TTY] n [TYT] n [YTT] $\notin Y$.

So, $Y$ is not a quasi-ideal of $T$.

(2) Take $Y=\left\{\left(\begin{array}{ll}1 & 0 \\ 0 & 0\end{array}\right)\right\}$ and $Q=\left\{\left(\begin{array}{ll}0 & 0 \\ 0 & 0\end{array}\right),\left(\begin{array}{ll}1 & 0 \\ 0 & 0\end{array}\right)\right\}$. Then $Y \subseteq Q$.

Again [TTQ] $\cup[T R T] \cup[Q T T] \cup[T T Q T T] \& Y$.

Since each of [TTY], [TYT], [YTT] contains $\left(\begin{array}{ll}0 & 0 \\ 0 & 0\end{array}\right)$, they are not contained in $Y$.

Hence $Y$ is neither a left, a lateral nor a right ideal of $T$.

Also [TTY] $\cap[T Y T] \cap[Y T T] \notin Y$.

So $Y$ is not a quasi-ideal of $T$.

Further [YTYTY] $\notin Y$ implies $Y$ is not a bi-ideal of $T$.

(3) Now we take $Y=\left\{\left(\begin{array}{ll}0 & 0 \\ 0 & 0\end{array}\right),\left(\begin{array}{ll}1 & 0 \\ 0 & 1\end{array}\right),\left(\begin{array}{ll}1 & 0 \\ 0 & 0\end{array}\right),\left(\begin{array}{ll}0 & 1 \\ 0 & 0\end{array}\right),\left(\begin{array}{ll}0 & 0 \\ 1 & 0\end{array}\right)\right\}$ and $Q=\left\{\left(\begin{array}{ll}0 & 0 \\ 0 & 0\end{array}\right)\right\}$ of $T$. '. ien $Y \notin Q$.

$$
[T T Q] \cup[T Q T] \cup[T T Q T T] \cup[Q T T]=\left\{\left(\begin{array}{ll}
0 & 0 \\
0 & 0
\end{array}\right)\right\} \subseteq Y \text {. }
$$

We find that $\left(\begin{array}{ll}0 & 0 \\ 0 & 0\end{array}\right) \in[T T Y],[T Y T]$ and $[Y T T]$.

But $\left(\begin{array}{ll}0 & 0 \\ 0 & l\end{array}\right) \notin Y$. So $\mathrm{Y}$ is either a left, a lateral nor a right ideal of $\mathrm{T}$. Similarly $\mathrm{Y}$ is neither a quasi nor a bi-ideal of $\mathrm{T}$.

THEOREM 3.9. Let $X, Y$ and $Z$ be three non-empty subsets of a ternary semigroup $T$ and $N=[X Y Z]$. Then $N$ is a bi-ideal of $T$ if one of the following conditions holds :

(1) $\mathrm{X}, \mathrm{Y} \subseteq \mathrm{Z}$ and $\mathrm{Z}$ is a bi-ideal of $\mathrm{T}$.

(2) $Y, Z \subseteq X$ and $X$ is a bi-ideal of $T$.

(3) $X, Z \subseteq Y$ and $Y$ is a bi-ideal of $T$.

(4) At least one of $X, Y, Z$ is a right, or a left or a lateral ideal of $T$.

PROOF. (1) $[\mathrm{NNN}] \subseteq[\mathrm{XYZ}[\mathrm{ZZZ}][Z Z Z]]$

$$
c[X Y[Z Z Z]] \subseteq N
$$

and $[N T N T N] \subseteq[X Y[Z T Z T Z]] \subseteq N$.

Sinilar proofs establish (2) and (3).

(4) Asssume $X$ is a right ideal of $T$. Then 


$$
\begin{aligned}
& [\text { NNN }] \subseteq[\text { X[TTT }][\text { TTT }] Y Z] \subseteq[\text { XTTYZ }] \subseteq N \\
& {[\text { NTNTN }] \subseteq[X[\text { TTT }][\text { TTT }] \text { TTYZ }] \subseteq[\text { XTTYZ }] \subseteq N .}
\end{aligned}
$$

Similar proofs can be given when $e i$ ther $X$ or $Y$ or $Z$ is a left, or a lateral or a right ideal of $T$.

DEFINITION 3.10 [7]. An element ' $t$ ' in a ternary semi group $T$ is sald to be regular if there exists $x, y$ in $T$ such that

$$
[\text { txtyt }]=t .
$$

If all the elements of $T$ are regular then it is said to be regular ternary semi group.

EXAMPLE 3.11. This example shows that there exists a ternary semi group while $T$ is not a regular ternary semi group such that $T$ has a minimal right, a minimal lateral and a minimal left ideal of $T$.

Let $\mathrm{T}=\{0, e, a, b\}$ be the ternary semi group under the operation $($ ), (given below in the table)

$\forall a, b, c \in T,[a b c]=a(b c)=(a b) c$.

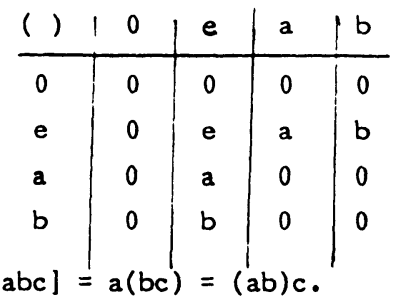

Hence $\{0\}$ is a minimal right, a minimal left and a minimal lateral ideal of $T$.

Since $a$ and $b$ are not the regular elements of $T$. Therefore $T$ is not a regular ternary semi group.

Now we use theorem 3.9 to give an example of a ternary semi group in which a bi-ideal is not a quasi-ideal.

EXAMPLE 3.12. Let $T$ be a ternary semi group such that $T$ is not regular, $X, Y, Z$ be respectively a minimal right, a minimal lateral and a minimal left ideal of $T$ satisfying the condition of 3.9. Thus $N=[X Y Z]$ is a bi-ideal of $T$. We will show that $N$ is not a quasi-ideal of $T$.

PROOF. $[X Y Z] \subseteq[X T T] \subseteq X,[X Y Z] \subseteq Y,[X Y Z]_{\_} \subset Z$. So, $[X Y Z] \subset X \cap Y \cap Z$ which is a minimal quasi-ideal of $T[7]$.

If we assume that $[X Y Z]$ is a quasi-ideal then $[X Y Z]=X \cap Y \cap Z$ which (by Sioson [7]) thus implies that $T$ is a regular ternary semi group. Hence it contradicts the hypothesis. So $[X Y Z]$ is not a quasi-ideal but bi-ideal by Theorem 3.9.

PROPOSITION 3.13. In a regular ternary semi group every bi-ideal is a quasiideal.

PROOF. Sioson [7] shows that a subset $Q$ of a regular ternary sem! group $T$ is a quasi-ideal if and only if 
[QTQTQ] $\cap$ [QTRTTQ] $\subseteq$ Q.

Since a bi-ideal of $T$, clearly satisfies the above condition, so we get the proof.

PROPOSITION 3.14. Let $C$ be a non-empty subset of a ternary semi group $T$ without identity. Then $C \cup[C C C] \cup[C T C T C]$ is the smallest bi-ideal of $T$ containing C.

PROOF. Let $x$ be any elemnt of $C \cup[C C C] \cup[C T C T C]$. Then either $x=x_{1}$ for $x_{1}$ in $C$ or $x=\left[\begin{array}{lll}c_{1} & c_{2} & c_{3}\end{array}\right] \in[O C C]$ for all $c_{i}$ in $C . i=1,2,3$ or $x=\left[\begin{array}{lll}c_{1} t_{1} c_{2} t_{2} c_{3}\end{array}\right]$

[CTCTC] for all $c_{i}$ in $C, i=1,2,3, t_{i}$ in $T, i=1,2$.

We will consider the elements of [CTCTC]. The other two cases will be done in similar manner. Let $x, y, z \in[$ [CICTC].

i.e., $x=\left[c_{1} t_{1} c_{2} t_{2} c_{3}\right], y=\left[c_{4} t_{3} c_{5} t_{4} c_{6}\right], z=\left[\begin{array}{lll}c_{7} t_{5} & c_{8} t_{6} c_{9}\end{array}\right], c_{1} \in C$, $\forall i=1,2, \ldots, 9, t_{i} \in T, \forall i=1,2, \ldots, 6$.

Then

$$
\begin{aligned}
{[x y z] } & =\left[\left[c_{1} t_{1} c_{2} t_{2} c_{3}\right]\left[c_{4} t_{3} c_{5} t_{4} c_{6}\right]\left[c_{7} t_{5} c_{8} t_{6} c_{9}\right]\right] \\
& =\left[c_{1}\left[\left[t_{1} c_{2} t_{2}\right]\left[c_{3} c_{4} t_{3}\right]\left[c_{5} t_{4} c_{6}\right]\right] c_{7}\left[t_{5} c_{8} t_{6}\right] c_{9}\right] \\
& =\left[c_{1} t_{7} c_{7} t_{8} c_{9}\right] \text { where } \\
t_{7} & =\left[\left(t_{1} c_{2} t_{2}\right]\left[c_{3} c_{4} t_{3}\right]\left[c_{5} t_{4} c_{6}\right]\right] \\
t_{8} & =\left[t_{5} c_{8} t_{6}\right]
\end{aligned}
$$

so $[\mathrm{xyz}] \in \mathrm{C} \cup[\infty \mathrm{C}]$ u [CTCTC].

Further, $\left[x t_{9} y t t_{10} z\right]=\left[\left[c_{1} t_{1} c_{2} t_{2} c_{3}\right] t_{9}\left[c_{4} t_{3} c_{5} t_{4} c_{6}\right] t_{10}\left[c_{7} t_{5} c_{8} t_{6} c_{0}\right]\right]$

$$
\text { where } \quad \begin{aligned}
& =\left[c_{1} t_{11} c_{6} t_{12} c_{9}\right] \\
t_{11} & =\left[\left(t_{1} c_{2} t_{2}\right]\left[c_{3} t_{9} c_{4}\right]\left[t_{3} c_{5} t_{4}\right]\right] \\
t_{12} & =\left[t_{10} c_{7}\left[t_{5} t_{8} t_{6}\right]\right], t_{9}, t_{10} \in T .
\end{aligned}
$$$$
=\left[c_{1}\left[\left[t_{1} c_{2} t_{2}\right]\left[c_{3} t_{9} c_{4}\right]\left[t_{3} c_{5} t_{4}\right]\right] c_{6}\left[t_{10} c_{7}\left[t_{5} c_{8} t_{6}\right]\right] c_{9}\right]
$$

Thus

$$
\left[\mathrm{xt}_{9} \mathrm{yt}_{10} z\right] \in \mathrm{C} \cup[\mathrm{OC}] \cup[\text { [CTCTC]. }
$$

Hence $C \cup[O C] \cup[C T C T C]$ is a bi-ideal of $T$ containing $C$.

Suppose there exists a bi-ideal $R$ of $T$ containing $C$ such that

$$
R \subseteq C \cup[C O C] \cup[C T C I C]
$$

Then $R$ being a bi-ideal implies that

$$
\mathrm{R} \subseteq \mathrm{C} \cup[\mathrm{CCC}] \cup[\mathrm{CTCTC}] \subseteq \mathrm{R} \cup[\mathrm{RRR}] \cup[\mathrm{RTRTR}] \subseteq \mathrm{R} .
$$

Thus $\mathrm{R}=\mathrm{C} \cup[C C C] \cup[C T C T C]$ is the smallest bi-ideal of $\mathrm{T}$ contain ing $\mathrm{C}$. 
ACKNOWLEDGEMENT. The authors are very thankful to the referee for his valuable comments and suggestions.

\section{REFERENCES}

1. DUDEK IZABELA, M. 'On Ternary Semi group Connected With Para Associative Rings' Riarche Mat. 35(1986), No. 2,191-203.

2. FEl LULLAER, R.B. 'Ternary Semi group Locally Homeomorphic'. Dokl. Akad.Nauk. SSSR 252(1980), No.5, 1063-1065.

3. KIM KI HANG and ROUSH FRED, W.'Ternary Semi group Associative on Each Pair of Eactor'. Simon Stevin 54(1980), No.2, 65-74.

4. LEHMER, D.H. 'A Ternary Analogue of Abelian Groups', Amer. Jr. of Maths. 59 (1932) $329-338$.

5. LOS, J. "On The Extending of Models I', Fundamenta Mathematicae 42(1955)38-54.

6. LYAPIN, E.S. 'Realization of Ternary Semi groups' (Russian) Modern Algebra, pp. 43-48 (Leningrad Univ.), Leningrad (1981).

7. SIOSON, F.M. 'Ideal Theory in Ternary Semi groups', Math. Japan 10(1965)63-84. 


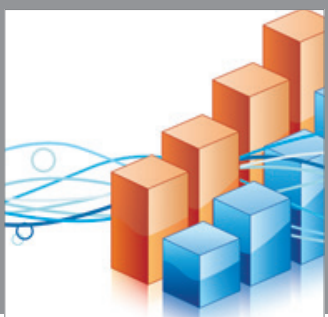

Advances in

Operations Research

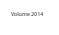

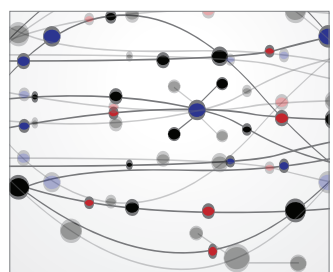

\section{The Scientific} World Journal
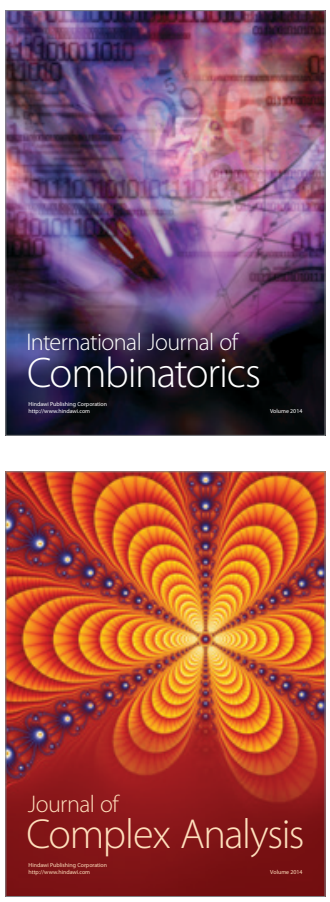

International Journal of

Mathematics and

Mathematical

Sciences
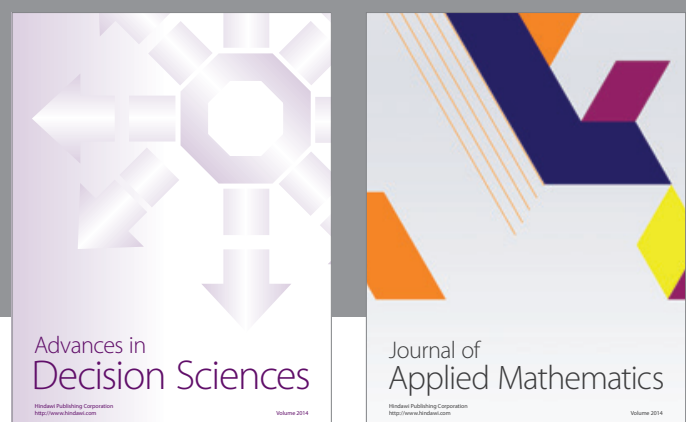

Journal of

Applied Mathematics
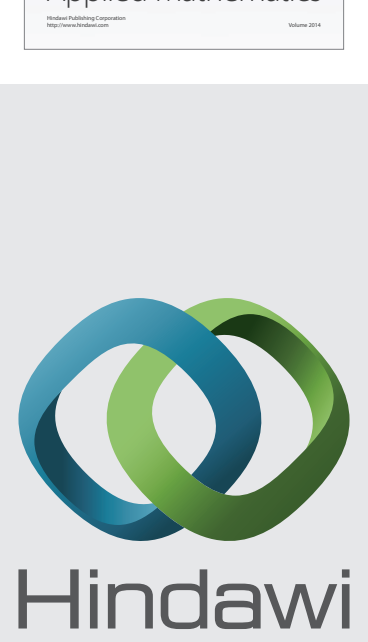

Submit your manuscripts at http://www.hindawi.com
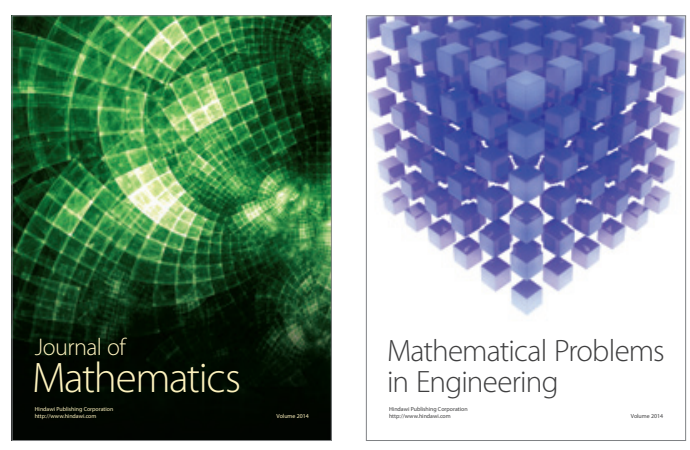

Mathematical Problems in Engineering
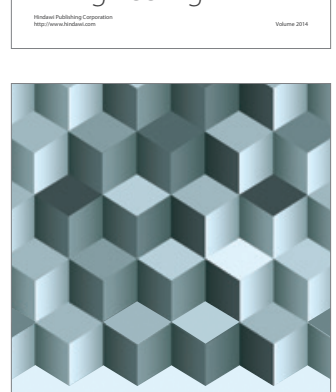

Journal of

Function Spaces
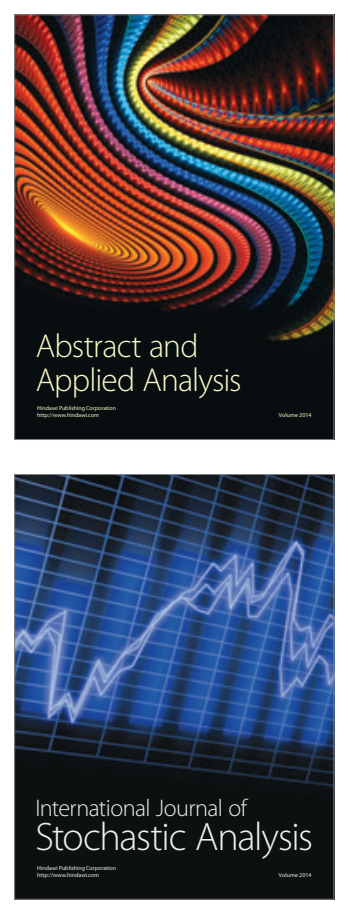

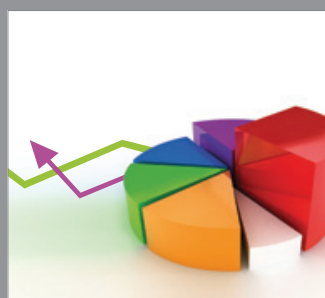

ournal of

Probability and Statistics

Promensencen
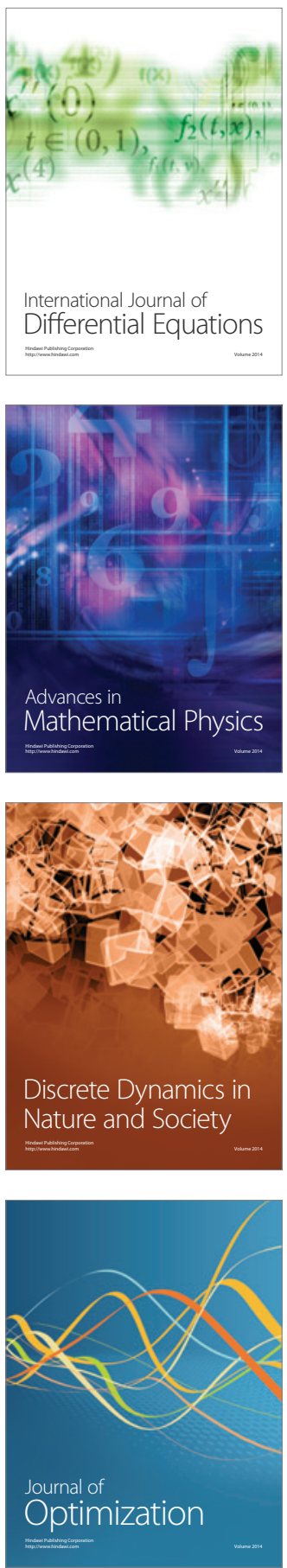